\title{
Asteroseismic estimate of helium abundance of 16 Cyg A, B
}

\author{
Kuldeep Verma ${ }^{1}$, João P. Faria ${ }^{2,3}$, H. M. Antia ${ }^{1}$, Sarbani Basu ${ }^{4}$, Anwesh Mazumdar ${ }^{5}$, Mário
} J. P. F. G. Monteiro ${ }^{2,3}$, Thierry Appourchaux ${ }^{6}$, William J. Chaplin ${ }^{7,8}$, Rafael A. García ${ }^{9}$, and Travis S. Metcalfe 8,10

1 Tata Institute of Fundamental Research, Homi Bhabha Road, Mumbai 400005, India

2 Centro de Astrofísica da Universidade do Porto, Rua das Estrelas, 4150-762 Porto, Portugal

3 Departamento de Física e Astronomia, Universidade do Porto, 4169-007 Porto, Portugal

4 Astronomy Department, Yale University, P. O. Box 208101, New Haven, CT 065208101, USA

5 Homi Bhabha Centre for Science Education, TIFR, V. N. Purav Marg, Mumbai 400088, India

6 Institut d'Astrophysique Spatiale, Université Paris XI-CNRS, F-91405 Orsay Cedex, France

7 School of Physics and Astronomy, University of Birmingham, B15 2TT, UK

8 Stellar Astrophysics Centre, Aarhus University, Ny Munkegade 120, DK-8000 Aarhus C, Denmark

9 Laboratoire AIM, CEA/DSM, CNRS, Université Paris Diderot, IRFU/SAp, Centre de Saclay, 91191 Gif-sur-Yvette Cedex, France

10 Space Science Institute, Boulder, CO 80301, USA

\begin{abstract}
The helium ionization zone in a star leaves a characteristic signature on its oscillation frequencies, which can be used to estimate the helium content in the envelope of the star. We use the oscillation frequencies of 16 Cyg A and B, obtained using 2.5 years of Kepler data, to estimate the envelope helium abundance of these stars. We find the envelope helium abundance to lie in the range 0.231-0.251 for 16 Cyg A and 0.2180.266 for 16 Cyg B.
\end{abstract}

A region of sharp variation in sound speed, known as acoustic glitch, introduces an oscillatory component, $\delta v$, in the frequencies of stellar oscillations as a function of the radial order, $n$, of the eigenmodes [1-3]. The frequency of this oscillatory signal depends on the acoustic depth of the glitch, and its amplitude depends on the extent of variation across the glitch in the sound speed or its derivatives. In the HeII ionization zone the adiabatic index, $\Gamma_{1}$ is depressed, which in turn affects the sound speed in that region, and consequently, contributes to an oscillatory signal. The amplitude of the oscillatory signal depends on the depression in $\Gamma_{1}$, and therefore on the amount of helium present there. This amplitude can be calibrated against stellar models of similar mass and effective temperature with varying helium abundance to estimate the envelope helium abundance $[4,5]$. We applied this technique to a binary system, $16 \mathrm{Cyg} \mathrm{A}$ and $\mathrm{B}$.

We estimated the amplitude of the helium signal using three different fitting methods: (1) fitted the second differences of the frequencies to Eq. (1) which was adapted from [6]; (2) fitted the residuals, obtained after subtracting the smooth component from the frequencies, to Eq. (2) [5]; and (3) fitted the frequencies directly to Eq. (3) with second derivative smoothing.

$$
\begin{aligned}
\delta^{2} v & =a_{0}+a_{1} v+\frac{b_{2}}{v^{2}} \sin \left(4 \pi \tau_{\mathrm{CZ}} v+\psi_{\mathrm{CZ}}\right)+c_{0} v e^{-c_{2} v^{2}} \sin \left(4 \pi \tau_{\mathrm{He}} v+\psi_{\mathrm{He}}\right) \\
\delta v & =A_{\mathrm{CZ}}\left(\frac{v_{r}}{v}\right)^{2} \cos \left(4 \pi \tau_{\mathrm{CZ}} v+\phi_{\mathrm{CZ}}\right)+A_{\mathrm{He}}\left(\frac{v_{r}}{v}\right) \sin \left(2 \pi \beta_{\mathrm{He}} v\right) \cos \left(4 \pi \tau_{\mathrm{He}} v+\phi_{\mathrm{He}}\right) \\
v_{n, l} & =\sum_{i=0}^{4} A_{l, i} n^{i}+\frac{A_{\mathrm{CZ}}}{v^{2}} \sin \left(4 \pi \tau_{\mathrm{CZ}} v+\phi_{\mathrm{CZ}}\right)+A_{\mathrm{He}} v e^{-c_{2} v^{2}} \sin \left(4 \pi \tau_{\mathrm{He}} v+\phi_{\mathrm{He}}\right)
\end{aligned}
$$

This is an Open Access article distributed under the terms of the Creative Commons Attribution License 4.0, which permits unrestricted use, distribution, and reproduction in any medium, provided the original work is properly cited. 

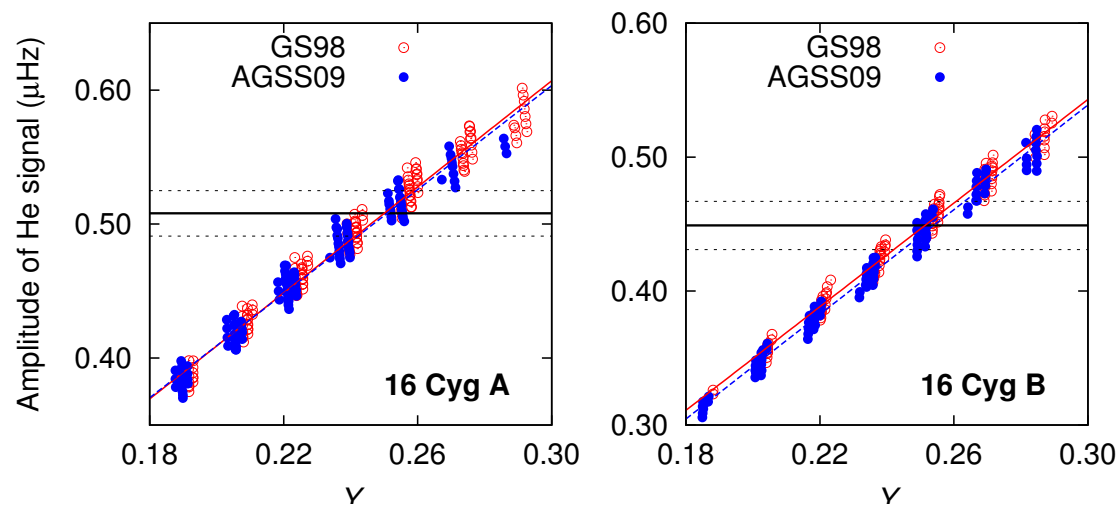

Fig. 1. Amplitude of the helium signal, obtained by fitting the frequencies to Eq. (3) and averaging the resulting amplitude over the frequency range used in the fit, as a function of helium abundance. The continuous horizontal line is the observed amplitude, with the dotted lines representing the $1 \sigma$ errors. The points are the results for the MESA models, while the red continuous and blue dashed lines show the straight line fit to these points.

Table 1. Helium abundance of 16 Cyg A and B.

\begin{tabular}{cccccccc}
\hline \hline & \multicolumn{3}{c}{16 Cyg A } & & \multicolumn{3}{c}{ 16 Cyg B } \\
\cline { 2 - 4 } \cline { 6 - 8 } Method & MESA GS98 & MESA AGSS09 & YREC GS98 & & MESA GS98 & MESA AGSS09 & YREC GS98 \\
\hline 1 & $0.238 \pm 0.009$ & $0.243 \pm 0.009$ & $0.231 \pm 0.009$ & & $0.263 \pm 0.012$ & $0.266 \pm 0.012$ & $0.257 \pm 0.009$ \\
2 & $0.239 \pm 0.021$ & $0.242 \pm 0.023$ & $0.236 \pm 0.016$ & & $0.218 \pm 0.013$ & $0.228 \pm 0.011$ & $0.219 \pm 0.009$ \\
3 & $0.250 \pm 0.009$ & $0.251 \pm 0.009$ & $0.249 \pm 0.009$ & & $0.251 \pm 0.010$ & $0.254 \pm 0.010$ & $0.255 \pm 0.009$ \\
\hline
\end{tabular}

The parameters in the above expressions were obtained by fits. We used three sets of calibrating models: two constructed with the MESA code [7] for two different metallicity mixtures (GS98 [8] and AGSS09 [9]) and one with the YREC code [10]. The model frequencies were fitted in the same manner and by using same modes and weights as the observations. Figure 1 shows the amplitude of the helium signal averaged over the frequency range used in the fit for both the stars. Clearly the amplitude is predominantly a function of current helium abundance in the models. The helium abundances obtained by calibrating the model amplitude with the observations are listed in Table 1 for both stars.

\section{References}

1. D.O. Gough, M.J. Thompson, in Advances in Helio- and Asteroseismology, edited by J. Christensen-Dalsgaard, S. Frandsen (1988), Vol. 123 of IAU Symposium, p. 155

2. S.V. Vorontsov, in Advances in Helio- and Asteroseismology, edited by J. Christensen-Dalsgaard, S. Frandsen (1988), Vol. 123 of IAU Symposium, p. 151

3. D.O. Gough, in Progress of Seismology of the Sun and Stars, edited by Y. Osaki, H. Shibahashi (1990), Vol. 367 of Lecture Notes in Physics, Berlin Springer Verlag, p. 283

4. S. Basu, A. Mazumdar, H.M. Antia, P. Demarque, MNRAS 350, 277 (2004)

5. M.J.P.F.G. Monteiro, M.J. Thompson, MNRAS 361, 1187 (2005)

6. G. Houdek, D.O. Gough, MNRAS 375, 861 (2007)

7. B. Paxton, L. Bildsten, A. Dotter, F. Herwig, P. Lesaffre, F. Timmes, ApJS 192, 3 (2011)

8. N. Grevesse, A. J. Sauval, Space Sci. Rev., 85, 161 (1998)

9. M. Asplund, N. Grevesse, A. J. Sauval, P. Scott, ARA\&A, 47, 48 (2009)

10. P. Demarque, D.B. Guenther, L.H. Li, A. Mazumdar, C.W. Straka, Ap\&SS 316, 31 (2008) 